\title{
Assessment of Organ Dose and Effective Dose from Head CT Examination in a Large Hospital in South-Southern Nigeria
}

\author{
R. I. Obed*, M. E. Ekpo \\ Department of Physics, University of Ibadan, Ibadan, Nigeria \\ Email: *rachelobed@yahoo.com
}

How to cite this paper: Obed, R.I. and Ekpo, M.E. (2018) Assessment of Organ Dose and Effective Dose from Head CT Examination in a Large Hospital in South-Southern Nigeria. International Journal of Medical Physics, Clinical Engineering and Radiation Oncology, 7, 431-437.

https://doi.org/10.4236/ijmpcero.2018.74036

Received: July 18, 2018

Accepted: September 25, 2018

Published: September 28, 2018

Copyright $\odot 2018$ by authors and Scientific Research Publishing Inc. This work is licensed under the Creative Commons Attribution International License (CC BY 4.0).

http://creativecommons.org/licenses/by/4.0/

\section{Open Access}

\begin{abstract}
The magnitude of radiation dose imparted to patients who underwent Head Computed Tomography examination in a large tertiary hospital in South-Southern Nigeria has been estimated. CT-ExPO dosimetry software was used to determine the effective and organ doses to the head region of 40 adult patients. Scanning parameters were retrieved from the CT monitor for both contrast-enhanced and non-contrast head CT examinations. The tube potential ranged from $100 \mathrm{kVp}$ to $120 \mathrm{kVp}$, while the mAs ranged from 127 $\mathrm{mAs}$ to $202 \mathrm{mAs}$. The mean values of the volume Computed Tomography Dose Index $\left(\mathrm{CTDI}_{\mathrm{vol}}\right)$ and Dose Length Product (DLP) for the contrast-enhanced Head CT examination were $166.4 \pm 39.6 \mathrm{mGy}$ and $3568.6 \pm$ $756.1 \mathrm{mGy} \cdot \mathrm{cm}$, respectively, while for the non-contrast examination they were $86.6 \pm 30.4 \mathrm{mGy}$ and $2102.3 \pm 870.3 \mathrm{mGy} \cdot \mathrm{cm}$, respectively. The effective doses were higher for the contrast-enhanced study than for the non-contrast study by a factor of 1.6. Results were compared with the European Union reference doses and other published data and were found to be higher. Doses to the organs which comprise of brain, red bone marrow, thyroid and eye lens were also estimated. The high variation in the doses in this study may be due to differences in imaging protocols such as large range of mAs and scan lengths and also the algorithm of the scanner.
\end{abstract}

\section{Keywords}

Computed Tomography Dose Index, Dose Length Product, Organ Doses, Effective Dose, CT-ExPO Software

\section{Introduction}

Ionizing radiation has been a major source of concern since the advent of $\mathrm{x}$-rays, 
mammography and computed tomography examinations. Computed Tomography (CT) examinations which use $\mathrm{x}$-rays to make detailed pictures of structures inside the body constitute the largest contribution to radiation exposure of the population thereby making diagnostic medical $\mathrm{x}$-rays the largest man-made source of ionizing radiation exposure [1]. Despite its large contribution to medical radiation exposure, the superiority of CT scanning over conventional radiography is based on the fact that

1) It has the ability to eliminate organ or structural super-imposition that usually occurs in conventional radiography.

2) As a digital modality, it has also the potential of producing diagnostic quality images with almost unlimited radiation doses since detectors are linear over long ranges.

3) Operator dependency palpable in ultrasonography is absent in CT, as CT images are reproducible.

4) It has better cortical bone definition and readily displays calcification unlike MRI [2].

Increase in the use of CT for diagnostic imaging is well documented; likewise the associated increase in radiation risk for patients undergoing CT investigations [3]. The increased use of ionizing radiation in diagnostic imaging, the rapidly expanding use of CT in the emergency setting, the introduction of multi-detector CT units and newly reported concerns related to the human consequences of low-level radiation exposure have revitalized a long-standing concern over the quantification and management of an individual's cumulative medical radiation exposure [4]. Studies have shown that many physicians, including radiologists have developed a misconception that the shorter imaging acquisition times have resulted in lower doses of radiation, when in fact, many times, the opposite is true. Body parts located in the central part of the body (chest/abdomen and pelvis) generally require higher levels of radiation exposure in order to obtain adequate imaging [5]. There is a small, theoretical risk of carcinogenesis attributable to low doses of ionizing radiation based on epidemiological evidence at higher doses and dose rates. Many studies have investigated the correlation between radiation exposure and cancer risk. Epidemiological studies, such as life span study of the atomic bomb survivors, medical studies and experimental animal research have established a relationship between exposure, cancer induction and cellular damage. However, there is lack of consensus on the effects of radiation at low doses and low dose rates, with the linear no threshold hypothesis being widely accepted and the basis of the international system of radiation protection [6]. Tissue reaction effects have precise radiation dose thresholds which induce radiation risks in relatively high doses [7].

Radiation doses associated with commonly used CT examinations resemble doses received by individuals in whom an increased risk of cancer had been documented [8] [9]. A single CT scan can deliver an equivalent radiation exposure and patients may receive multiple CT scans over time [10]. Even though the 
risk to an individual patient may be small, the increasingly large number of people exposed, coupled with the increasingly high exposure per examination could translate into many cases of cancer resulting directly from the radiation exposure from CT. It is important to understand how much radiation medical imaging delivers, so this potential for harm can be balanced against the potential for benefit. This is particularly important as the threshold for using CT has declined, and CT is increasingly being used among healthy individuals, where the potential for carcinogenesis could outweigh its diagnostic value [11].

The head CT scan has been the most common CT examination performed in Europe (30\%-40\%), for more than a decade, and it also contributes significant total collective effective dose to the population [12]. Likewise in Nigeria, head CT examinations is the commonest CT examination performed in most CT centres. The significant radiation dose delivered to superficial radiosensitive organs, such as lens of the eye, unavoidably irradiated during radiological procedures of the head, is also a concern. Even though, there are non-ionising imaging modalities, such as magnetic resonance imaging that are currently used for imaging of the head, CT imaging continues to be on the increase due to its wide availability and clinical application. It is impractical to directly measure the radiation dose absorbed by individual patients even when the radiation emitted by a machine is precisely known. Instead, radiation exposure may be quantified using various methods. The evaluation of effective dose, $\mathrm{E}$, in diagnostic radiology is an important tool for risk estimates from medical exposures. This is one of the most frequently reported measurements [6]. Further, effective dose allows comparison across the different types of CT studies and also between CT and other imaging tests, facilitating comparison of $\mathrm{CT}$ to the most common radiology examinations that patients undergo. The effective dose accounts for the amount of radiation to the exposed organs, and each organs sensitivity to developing cancer from radiation exposure. Hence, the benefit derived by patients should far outweigh the associated risk when the imaging is properly conducted. This study, therefore, aims to assess how much radiation exposure has been imparted to patients during head CT examinations at a large tertiary hospital in the South-Southern region of Nigeria.

\section{Materials and Methods}

This study involved 40 adult patients of mixed gender ( 25 males and 15 females) referred for head CT scan at a large tertiary hospital in the South-Southern region of Nigeria. Their ages ranged from 18 to 82 years with a mean of $48 \pm 17.6$ years. Ethical approval was obtained from the local ethics committee of the hospital. Computed tomography examination of the head was the most frequently requested examination at this hospital, therefore, scan-specific parameters for this examination were collected. Displayed CT console measurements of CTDI and DLP along with records of each patient's scanning parameters were obtained from the Toshiba 16-slice Aquillion CT equipment used at the hospital. Organ 
and effective doses were estimated using CT-ExPO (version 2.5) dosimetry software. The organ doses to the brain, eye lens, red bone marrow (RBM) and thyroid were estimated from the head CT examination. The prospective study comprising of contrast-enhanced and non-contrast Head CT examinations lasted for 3 months. The CT-ExPO software is based on Monte Carlo data sets and has a unique feature of organ dose calculation for specific gender. For the head scan region, the following exposure parameters were obtained from the CT scanner used: tube voltage, tube current, scan range, rotation time, spiral pitch, and collimation. The tube potential ranged from $100 \mathrm{kVp}$ to $120 \mathrm{kVp}$, while the $\mathrm{mAs}$ ranged from $127 \mathrm{mAs}$ to $202 \mathrm{mAs}$. The mean exposure parameters used for the examinations under study are presented in Table 1.

\section{Results and Discussion}

The demographic and dose characteristics of patients are presented in Table 2. The mean values of the volume Computed Tomography Dose Index (CTDI ${ }_{\mathrm{vol}}$ ) and Dose Length Product (DLP) for the contrast-enhanced Head CT examination were $166.4 \pm 39.6 \mathrm{mGy}$ and $3568.6 \pm 756.1 \mathrm{mGy} \cdot \mathrm{cm}$, respectively, while for the non-contrast they were $86.6 \pm 30.4 \mathrm{mGy}$ and $2102.3 \pm 870.3 \mathrm{mGy} \cdot \mathrm{cm}$, respectively. Table 3 compares the CTDI ${ }_{\mathrm{vol}}$ and DLP from this study with published data and European Union (EU) reference doses for head CT examinations (no contrast). The CTDI ${ }_{\text {vol }}$ and DLP were higher than the EU values by factors of 1.4 and 2, respectively. Table 4 shows the comparison of estimated effective doses for contrast-enhanced and non-contrast Head CT examinations in this study with published data and EC reference values. The effective doses were higher for the contrast-enhanced study than for the non-contrast study by a factor of 1.6. Comparisons of the mean effective doses from this study with previous studies, in a large tertiary hospital in south-western, Nigeria showed a large difference up to a factor of 3.6 [13]. The effective dose from the non-contrast CT was also higher than EU reference value by a factor of 5 .

Doses to the organs which comprise of brain, red bone marrow, thyroid and eye lens were $83.2 \mathrm{mGy}, 7.9 \mathrm{mGy}, 5.2 \mathrm{mGy}$, and $107.6 \mathrm{mGy}$, respectively for contrast-enhanced studies whereas, for non-contrast studies they were 79.8 $\mathrm{mGy}, 7.7 \mathrm{mGy}$, 5.0 $\mathrm{mGy}$, and $105.8 \mathrm{mGy}$, respectively. The organ doses to the

Table 1. Mean Head CT scan technique parameters in the hospital.

\begin{tabular}{cc}
\hline Exposure Parameters & Mean \\
\hline Tube voltage & $119 \mathrm{kVp}$ \\
Tube current x time & $195 \mathrm{mAs}$ \\
Pitch & 0.5 \\
Rotation time & $0.75 \mathrm{~s}$ \\
Scan length & $20 \mathrm{~cm}$ \\
Slice thickness & $4.9 \mathrm{~mm}$ \\
\hline
\end{tabular}


Table 2. Demographic and dose characteristics of patients.

\begin{tabular}{|c|c|c|c|c|c|c|}
\hline \multirow{2}{*}{$\begin{array}{c}\text { Examination } \\
\text { Type }\end{array}$} & \multirow{2}{*}{ No. of patients } & \multicolumn{2}{|c|}{ Patient age (years) } & \multicolumn{3}{|c|}{ Effective Dose (mSv) } \\
\hline & & Range & Mean & Range & Mean & $75^{\text {th }}$ percentile \\
\hline Contrast-enhanced & 25 & & & $11.7-26.0$ & $15.3 \pm 3.2$ & 16.1 \\
\hline No contrast & 15 & $18-82$ & $48 \pm 17.6$ & $2.2-19.3$ & $9.2 \pm 3.8$ & 10.0 \\
\hline
\end{tabular}

Table 3. Comparison of mean $\mathrm{CTDI}_{\mathrm{vol}}(\mathrm{mGy})$ and DLP $(\mathrm{mGy} \cdot \mathrm{cm})$ for no contrast examination with previous studies and EU reference values.

\begin{tabular}{cccccc}
\hline Dose indices & This Study & {$[13]$} & {$[12]$} & {$[14]$} & {$[15]$} \\
\hline CTDI $_{\text {vol }}$ & 86.6 & 73.5 & 77 & 52.2 & 60 \\
DLP & 2102.3 & 1898 & 985 & 841.5 & 1050 \\
\hline
\end{tabular}

Table 4. Comparison of effective dose E (mSv) with previous studies and EC reference value.

\begin{tabular}{cccccc}
\hline Examination Type & This Study & {$[16]$} & {$[13]$} & {$[17]$} & {$[15]$} \\
\hline Contrast-enhanced & 16.1 & 6.1 & - & & - \\
No contrast & 10.0 & 3.1 & 2.8 & 1.8 & 2.0 \\
\hline
\end{tabular}

brain and eye lens were factors of 2.9 and 1.9 higher, respectively, when compared with values of organ doses obtained by [18]. The doses to the brain and eye lens during head CT examination in this large tertiary hospital appear to deserve attention. The high variation in doses in this study may be due to differences in imaging protocols such as large range of $\mathrm{mAs}$ and scan lengths and also the algorithm of the scanner. These findings strongly justify the need for immediate optimization of protocols at the hospital.

\section{Conclusion}

Organ doses and effective doses from head CT examinations in a large tertiary hospital in South-Southern Nigeria have been determined using the CT-ExPO dosimetry software. A significant variation was observed between this study and published data including EC reference values. An immediate intervention modality to harmonize CT protocols has become pertinent in Nigeria in order to optimize the doses delivered to patients during CT examinations. Periodic patient dosimetry evaluation and quality assurance programme should be an essential part of every radiological department of a hospital.

\section{Acknowledgements}

The authors would like to acknowledge with much gratitude the kind assistance of the Radiographers and Radiologists of the hospital during data collection The authors wish to also acknowledge the support of the Chief Medical Physicist, Maggiore Hospital, Trieste, Italy in collaboration with the Abdus Salam International Centre for Theoretical Physics (ICTP), Trieste, Italy for the purchase of 
the dosimetry software.

\section{Conflicts of Interest}

The authors declare no conflicts of interest regarding the publication of this paper.

\section{References}

[1] National Council on Radiation Protection and Measurements (2009) Ionizing Radiation Exposure of the United States. National Council on Radiation Protection Report No. 160, National Council on Radiation Protection and Measurement, Bethesda, MD.

[2] George, T., Rayan Babu, K.N. and Thomas, P.T. (2016) Reliability of Ultrasonography in the Diagnosis of Acute Appendicitis. International Surgery Journal, 3, 59-61. https://doi.org/10.18203/2349-2902.isj20151509

[3] Rehani, M.M. and Berry, M. (2000) Radiation Doses in Computed Tomography. BMJ, 320, 593-594. https://doi.org/10.1136/bmj.320.7235.593

[4] Berrington de Gonzalez, A. and Darby, S. (2004) Risk of Cancer from Diagnostic X-Rays: Estimates for the UK and 14 Other Countries. Lancet, 363, 345-351. https://doi.org/10.1016/S0140-6736(04)15433-0

[5] Dehn, T.G. (2007) Ionizing Radiation Exposure from Radiologic Imaging: The Issue of What We Can Do. https://hmsa.com/portal/provider/Radiation_Safety_Provider_Edu_4pgs.pdf

[6] International Commission on Radiological Protection (ICRP) Publication (2007) The 2007 Recommendations of the International Commission on Radiological Protection. Annals of the ICRP, 37, 1-332.

[7] International Commission on Radiological Protection (ICRP) Publication (2007) Managing Patient Dose in Multi-Detector Computed Tomography (MDCT). Annals of the ICRP, 37, 1-63.

[8] Pierce, D.A. and Preston, D.L. (2000) Radiation-Related Cancer Risks at Low Doses among Atomic Bomb Survivors. Radiation Research, 154, 178-186. https://doi.org/10.1667/0033-7587(2000)154[0178:RRCRAL]2.0.CO;2

[9] Preston, D.L., Pierce, D.A., Shimizu, Y., Ron, E. and Mabuchi, K. (2003) Dose Response and Temporal Patterns of Radiation-Associated Solid Cancer Risks. Health Physics, 85, 43-46. https://doi.org/10.1097/00004032-200307000-00010

[10] Sodickson, A., Baeyens, P.F., Andriole, K.P., Prevedello, L.M., Nawfel, R.D., Hanson, R. and Khorasani, R. (2009) Recurrent CT, Cumulative Radiation Exposure, and Associated Radiation-Induced Cancer Risks from CT of Adults. Radiology, 251, 175-184. https://doi.org/10.1148/radiol.2511081296

[11] Smith-Bindman, R., Lipson, J., Marcus, R. Kim, K., Mahesh, M., Gould, R., Berrington de Gonzalez, A. and Miglioretti, D.L. (2009) Radiation Dose Associated with Common Computed Tomography Examinations and the Associated Lifetime Attributable Risk of Cancer. Archives of Internal Medicine, 169, 2078-2086. https://doi.org/10.1001/archinternmed.2009.427

[12] Garba, I., Engel-Hills, P., Davidson, F. and Tabari, A.M. (2015) Computed Tomography Dose Index for Head CT in Northern Nigeria. Radiation Protection Dosimetry, 165, 98-101.

[13] Ogbole, G.I. and Obed, R.I. (2014) Radiation Doses in Computed Tomography: Need for Optimization and Application of Dose Reference Levels in Nigeria. West 
African Journal of Radiology, 21, 1-6.

[14] Abdulkadir, M.K., Schandorf, C. and Hasford, F. (2016) Determination of Computed Tomography Diagnostic Reference Levels of North-Central Nigeria. The Pacific Journal of Science and Technology, 17, 341-349.

[15] European Commission (1999) Radiation Protection 109. Guidance on Diagnostic Reference Levels (DRLs) for Medical Exposures. Office for Official Publications of the European Communities, Luxembourg.

[16] Adejoh, T., Christian, N.C., Nkubil, F.B. and Dlama, J.Z. (2015) Effective Dose Levels from Computed Tomography of the Head during Contrast Studies in Nigeria. Health, 7, 915-919.

[17] Osei, E.K. and Darko, J. (2013) A Survey of Organ Equivalent and Effective Doses from Diagnostic Radiology Procedures. ISRN Radiology, 2013, 9.

[18] Akpochafor, M.O., Omojola, A.D., Habeebu, M.Y., Ezike, J.C., Adeneye, S.O., Ekpo, M.E., Aweda, M.A., Opadele, A.E. and Orotoye, T.A. (2018) Computed Tomography Organ Dose Determination Using Impact Simulation Software: Our Findings in South-West Nigeria. Eurasian Journal of Medicine and Oncology, 2, 165-172. 